\title{
155. Remarks on Some Riemann Surfaces
}

\author{
By Kikuji Matsumoto \\ Mathematical Institute, Nagoya University \\ (Comm. by K. Kunugi, M.J.A., Dec. 12, 1958)
}

1. In the theory of meromorphic functions, it is important to investigate the properties of covering surfaces generated by their inverse functions. For this purpose, the study of properties of a noncompact region of a Riemann surface is useful.

Recently Kuramochi [4] gave interesting results for non-compact regions of some Riemann surfaces and these results were extended by Constantinescu and Cornea [1] and himself [5]. On the other hand, the method given by Heins [2] is also expected to contribute for the same purpose. So, in this note, we shall investigate some properties of covering surfaces using Kuramochi's results and Heins' method. Here we shall omit the details which will appear elsewhere.

2. Let $R_{1}$ and $R_{2}$ be two Riemann surfaces which do not belong to $O_{G}$, and let $f$ be a conformal mapping of $R_{1}$ into $R_{2}$. We denote by $\mathscr{S}_{R_{1}}$ and $\mathscr{H}_{R_{2}}$ Green functions of $R_{1}$ and $R_{2}$ respectively. Then, holds the equality

$$
\mathscr{S}_{R_{2}}(f(p) ; q)=\sum_{f(r)=q} n(r) \mathscr{G}_{R_{1}}(p ; r)+u_{q}(p),
$$

where $n(r)$ is the multiplicity of $f$ at $r \in R_{1}$, and $u_{q}(p)$ is the greatest harmonic minorant of $\mathscr{S}_{R_{2}}(f(p) ; q)$ on $R_{1}$.

Generally, a positive harmonic function is representable uniquely by the sum of a positive quasi-bounded harmonic function and a positive singular harmonic function (Parreau [7]). Heins [2] proved that $u_{q}(p)$ is quasi-bounded except for a set of $q$ of capacity zero and that the quasi-bounded component of $u_{q}(p)$ is either positive on $R_{1} \times R_{2}$ or constantly zero.

According to Heins [2], we say that $f$ is of type-Bl if the second alternative occurs for $f$.

Now, let $R_{1}$ and $R_{2}$ be arbitrary Riemann surfaces, and let $f$ be a conformal mapping of $R_{1}$ into $R_{2}$. We shall say that $f$ is of type-Bl at $q \in R_{2}$ provided that there exists a simply-connected Jordan region $\Omega$ satisfying: (1) $q \in \Omega \subset R_{2}$, (2) $f^{-1}(\Omega) \neq \phi$ and (3) for each component $\Delta$ of $f^{-1}(\Omega)$, the restriction $f_{\Delta}$ of $f$ to $\Delta$ is of type-Bl considering $f_{\Delta}$ as to be a conformal mapping of $\Delta$ into $\Omega$. We shall say that $f$ is locally of type- $\mathrm{Bl}$ if $f$ is of type- $\mathrm{Bl}$ at each point of $R_{2}$.

For simplicity, we shall call a non-compact or compact domain on a Riemann surface $R$ a subregion on $R$ when its relative boundary with respect to $R$ consists of at most an enumerable number of 
analytic curves being compact or non-compact and clustering nowhere in $R$. Then, we get the following:

Theorem 1. Let $R_{1}$ and $R_{2}$ be arbitrary Riemann surfaces, and let $f$ be a conformal mapping of $R_{1}$ into $R_{2}$. Then, $f$ is locally of type-Bl if and only if, for any compact subregion $\Omega$ on $R_{2}$ (we suppose that $\Omega$ has at least one exterior point when $R_{2}$ is compact), each component of $f^{-1}(\Omega)$ belongs to $S O_{H B}$.

3. According to Constantinescu and Cornea [1], we denote by $O_{H B_{n}}\left(O_{H D_{n}}\right)(1 \leqq n \leqq \infty)$ the class of Riemann surfaces, the ideal boundary of which consists of at most $n \mathrm{HB}$ - (maximal $\mathrm{HD}$-) indivisible sets in their sense. These classes are the same ones considered by Kuramochi [5]. In fact, as Constantinescu and Cornea proved, $O_{H B_{n}}\left(O_{H D_{n}}\right)(1 \leqq n$ $<\infty$ ) coincides with the class of Riemann surfaces on which there exist at most $n$ number of linearly independent bounded (Dirichletbounded) harmonic functions.

Heins [3] introduced a class $O_{L}$ of Riemann surfaces, on which there exists no non-constant single-valued Lindelöfian meromorphic function. Here, we say a conformal mapping of a Riemann surface $R_{1}$ into another Riemann surface $R_{2}$ is Lindelöfian if

$$
\sum_{f(r)=q} n(r) \mathscr{S}_{R_{1}}(p ; r)<+\infty
$$

for $f(p) \neq q$. It was proved by Heins that the inclusion-relation

$$
O_{H B_{1}}=O_{H B} O_{L} O_{A B}
$$

holds and that, for the class of Riemann surfaces with finite genus,

holds.

$$
O_{G}=O_{H B}=O_{L}
$$

Theorem 2 (Kuramochi). If a Riemann surface $R$ belongs to $O_{H B_{n}}-O_{G}(1 \leqq n \leqq \infty)$ and the subregion $G$ on $R$ does not belong to $S O_{H B}$, then $G$ belongs to $O_{L}$.

Kuroda [6] introduced a class $O_{A B}^{0}$ of Riemann surfaces whose all subregions belong to $S O_{A B}$ and proved that each Riemann surface belonging to $O_{A B}^{0}$ has Iversen property, and the inclusion-relation

$$
O_{I H B} O_{A B}^{0} O_{A B}
$$

holds and that, for the class of Riemann surfaces with finite genus,

$$
O_{G}=O_{H B} O_{A B}^{0} \subset O_{A B} \text {. }
$$

Noticing Theorem 2, we can verify the following:

$$
O_{L} \text { ‡ } O_{A B}^{0} \text { and } O_{L} \text { ‡ } O_{H D} \text {. }
$$

Theorem 3 (Kuramochi). If a Riemann surface $R$ belongs to $O_{H D_{n}}-O_{G}(1 \leqq n \leqq \infty)$ and the subregion $G$ on $R$ does not belong to $S O_{H D}$, then $G$ belongs to $O_{A D}$.

4. Here we shall state some results which are deduced from Theorems 1 and 2. 
Theorem 4. If a Riemann surface $R$ belongs to $O_{H B_{n}}(1 \leqq n \leqq \infty)$, then any non-constant single-valued meromorphic function $f$ on $R$ is locally of type-Bl.

Corollary. Let $R$ be Riemann surface belonging to $O_{H B_{n}}(1 \leqq n$ $\leqq \infty)$, and let $\Phi$ be the covering surface of the $w$-plane generated by a non-constant single-valued meromorphic function $f$ on $R$. Then every connected piece $\Phi_{\delta}$ of $\Phi$ on any disc $\delta$ on the $w$-plane covers the same times each point of $\delta$ except at most an $F_{\sigma}$-set of capacity zero.

Theorem 5. Let $R$ be a Riemann surface belonging to $O_{H B_{n}}$ $(1 \leqq n \leqq \infty)$ and let $G$ be a subregion on $R$ not belonging to $\mathrm{SO}_{H B}$. Then the cluster set of any non-constant single-valued meromorphic function $f$ on $G$ at the ideal boundary of $G$ is total, and the range of values of $f$ contains all values of the w-plane except for at most an $F_{\sigma}$-set of capacity zero.

5. Here we concern with the subsurface on Riemann surfaces of the class $O_{H D_{n}}$.

Theorem 6. Let $f$ be a non-constant single-valued meromorphic function on a Riemann surface $R$. If there exist a point $w_{0}$ and the sequence of Jordan regions $\Omega_{i}$ of the w-plane such that $\Omega_{i} \supset \bar{\Omega}_{i+1}$, $\bigwedge_{i=1}^{\infty} \Omega_{i}=w_{0}$, and that, for each $i$, at least one component $\Delta_{i}$ of $f^{-1}\left(\Omega_{i}\right)$ does not belong to $S O_{H D}$, then $R$ does not belong to $O_{H D}$.

Theorem 7. Let $R$ be a Riemann surface belonging to $O_{H D_{n}}$ $(1 \leqq n \leqq \infty)$, let $\Phi$ be the covering surface of the $w$-plane generated by a non-constant single-valued meromorphic function $f$ on $R$, and let $\Phi_{\rho}$ be a connected piece of $\Phi$ on $\left|w-w_{0}\right|<\rho$. If the area of $\Phi_{\rho}$ is finite, then the restriction $f_{\rho}$ of $f$ to the component $\Delta_{\rho}$ of $f^{-1}\left(\left|w-w_{0}\right|<\rho\right)$ corresponding to $\Phi_{\rho}$ is of type-Bl of $\Delta_{\rho}$. So, $\Phi_{\rho}$ covers each point of $\left|w-w_{0}\right|<\rho$ the same times except at most a closed set of capacity zero and $\Phi_{\rho}$ is finitely sheeted.

It is evident that this theorem implies Kuramochi's result (Theorem 12 in [5]).

\section{References}

[1] C. Constantinescu and A. Cornea: Über den idealen Rand und einige seiner Anwendungen bei Klassifikation der Riemannschen Flächen, Nagoya Math. J., 13, 169-233 (1958).

[2] M. Heins: On the Lindelöfian principle, Ann. Math., 61, 440-473 (1955).

[3] M. Heins: Lindelöfian maps, Ann. Math., 62, 418-445 (1955).

[4] Z. Kuramochi: On the behaviour of analytic functions on abstract Riemann surfaces, Osaka Math. J., 7, 109-127 (1955).

[5] Z. Kuramochi: On the ideal boundaries of abstract Riemann surfaces, Osaka Math. J., 10, 83-102 (1958). 
[6] T. Kuroda: On analytic functions on some Riemann surfaces, Nagoya Math. J., 10, 27-50 (1956).

[7] M. Parreau: Sur les moyennes des fonctions harmoniques et analytiques et la classification des surfaces de Riemann, Thèsis, Paris (1952). 